\title{
Facilitation of Attention to Number and Conservation of Number
}

\author{
Patricia H. Miller and Kirby A. Heller \\ University of Michigan
}

\begin{abstract}
This study examined the relation between number conservation and attention to number, density, and length or area in kindergarteners (age 5 years, 8 months) and third graders (age 8 years, 11 months). Attention was assessed by the dimensional preference technique, using triads. Both the attention and conservation tasks included stimulus factors known to facilitate or hinder conservation: small or large numbers of objects, nonlinear or linear arrays, and trials ordered from easiest to hardest, hardest to easiest, or randomly. The number of objects and type of array affected both tasks similarly and there was an order effect in the attention task. There was an increase in attention to number from kindergarten nonconservers to kindergarten conservers to third-graders. Possible developmental interactions between attention and conservation were suggested.
\end{abstract}

It has been suggested that young children have several definitions of quantity (e.g., length, density, true number) at the same time (Gelman, 1972; Zimiles, 1966). Which definition is used depends partly on the situation. Miller, Heldmeyer, and Miller (1975) found that the addition of perceptual supports in a number conservation task was associated with an increase in the percentage of children giving conservation answers from $41 \%$ for the hardest trial to $77 \%$ for the easiest one. The facilitating factors were a small number of objects, an increased salience of one-to-one correspondence cues, random configurations rather than lines, and receiving the trials in order from easiest to hardest. This order effect suggested that children developed a "set" for number on the early trials and maintained this set on trials in which they otherwise would have given nonconservation answers. Others have also found the facilitating effect of perceptual supports for correspondence (Whiteman \& Peisach, 1970) and a small number of objects (Baron, Lawson, \& Siegel, 1975; Gelman, 1972; Lawson, Baron, \& Siegel, 1974; Smither, Smiley, \& Rees, 1974; Winer, 1974). Thus, during the preschool and early elementary school years, a child's choice of a definition of quantity often can be manipulated by the particular assessment procedures.

This study was supported by University of Michigan Rackham Faculty Research Grant 984-360222. The authors are grateful to Abbot School for participating in the study. Requests for reprints should be sent to Patricia H. Miller, Department of Psychology, University of Michigan, Ann Arbor, MI 48109. 
What process underlies the impact of these stimulus variables? Several possibilities come to mind; ease of counting the objects, amount of information to process, use of estimators of quantity (Gelman, 1972), and attention. Recent research suggests that attention to stimulus dimensions is related to conservation performance. Gelman (1969) found that nonconservers who were trained to ignore irrelevant dimensions (e.g., length in a number conservation problem) and attend to relevant ones (number) were then able to conserve on standard conservation tasks. Miller (1973) compared attentional preferences and performance on a liquid conservation task. To assess attention to a particular dimension, she used the method of triads in which any two arrays were the same on one dimension and different on two others. A child's choice of any pair as being the "same" thus indicated which dimension was most salient for that trial. Preferences were primarily for liquid height in nonconservers, quantity in third-grade conservers, and, surprisingly, height in kindergarten conservers. Using a similar paradigm to study attention and conservation of number, Henry (1971) found that kindergarten conservers attended to number more than kindergarten nonconservers but that the conservers still preferred length to number. Lawson et al., (1974) asked preschoolers which of two static rows had more dots and which was longer. They found consistent biases toward number or length, regardless of the question asked. For example, a child with a bias toward number correctly answered about number but incorrectly indicated that rows with more objects were longer. These biases can be reversed through training (Baron et al., 1975). Lawson et al. also found that most of the children who gave a conservation answer on the one trial with a transformation used the number cue on the static arrays. Unlike the Miller and Henry studies, there was no "free attention" situation; children were directly asked about length and number. Nevertheless, the study suggests that some preschoolers will consistently use number cues in several similar situations.

As a further step toward specifying the relationship between attention and conservation, the present study examined whether stimulus variables known to facilitate conservation (e.g., Miller et al., 1975) have similar effects on attention to number and conservation of number. The variables were number of objects, linear arrays versus nonlinear arrays with objects placed randomly, and the order of the trials, beginning with the trial in which number is most salient and ending with the trial in which number is least salient, the opposite order, and a random order. Similar effects of these variables on both attention and conservation would lend further support to the hypothesis that differences in attentional preferences are related to conservation performance and that facilitative factors are effective because they direct attention to the relevant dimension. 
In addition to kindergarten nonconservers and conservers, thirdgraders were included. A comparison of conservers of two ages tests the prediction that the more stable the conservation concept, the more attention there is to number (Henry, 1971; Miller, 1973).

\section{METHOD}

\section{Subjects}

The subjects were 45 kindergarten boys, 41 kindergarten girls, 10 third-grade boys, and 8 third-grade girls from a predominantly white, middle-class, elementary school in Ann Arbor, Michigan. The mean ages were 5 years, 8 months for the kindergarteners and 8 years, 11 months for the third-graders. Eleven additional kindergarteners were rejected because they failed to meet the criteria defined below.

\section{Overview}

The attention task was a dimensional preference task based on the method of triads (e.g., Suchman, 1966). There were 14 trials, each having three cards with objects on them. The trials differed along the following dimensions: (a) number of objects on the three cards $(3,3,6$; $3,6,6 ; 6,6,9 ; 6,9,9 ; 6,6,12 ; 6,12,12$ ), and (b) type of array (objects arranged in a line on the card or scattered randomly all over the card). On each trial the child chose the two cards that seemed most alike to him. Attention to number, density, or length, in the case of linear displays, or area, in the case of objects placed randomly, was inferred from this choice.

The conservation task consisted of five trials which varied in whether arrays of four or eight objects were used and whether the objects were in lines or placed randomly in a nonlinear display.

\section{Materials}

Attention task. The stimuli were black-and-white drawings of identical birds "in flight." Their asymmetrical shape gave the desired impression of random placement on trials in which the objects were not presented in lines. The birds, each $3 \mathrm{~cm}$ long, were pasted on blue poster board rectangles which were slightly larger than the largest array in the trial.

The construction of triads was based on three dimensions: number, density, and either length (with rows of objects) or area (with random placement of objects). On each trial, any two of the three cards were the same on one dimension and different on the other two (see Table 1). For clarity of presentation, the diameters rather than the areas of the nonlinear arrays are described in Table 1.

Each child received four trials with each set of numbers $(3-6,6-9$, 
TABLE 1

Description of Stimuli on AtTention Trials

\begin{tabular}{|c|c|c|c|c|c|}
\hline \multirow[b]{2}{*}{ Trials } & \multirow[b]{2}{*}{ Dimensions } & \multirow{2}{*}{$\begin{array}{l}\text { Order of } \\
\text { trials }^{a}\end{array}$} & \multicolumn{3}{|c|}{ Card } \\
\hline & & & 1 & 2 & 3 \\
\hline \multirow[t]{3}{*}{1} & Number & 10 & 3 & 6 & 6 \\
\hline & Length & & 20 & 20 & 40 \\
\hline & Density & & 9 & 18 & 9 \\
\hline \multirow[t]{3}{*}{2} & Number & 2 & 3 & 3 & 6 \\
\hline & Length & & 20 & 40 & 40 \\
\hline & Density & & 9 & 18 & 9 \\
\hline \multirow[t]{3}{*}{3} & Number & 6 & 3 & 6 & 6 \\
\hline & Diameter & & 10 & 10 & 15 \\
\hline & Density & & 7 & 4 & 7 \\
\hline \multirow[t]{3}{*}{4} & Number & 1 & 3 & 6 & 6 \\
\hline & Diameter & & 10 & 15 & 15 \\
\hline & Density & & 7 & 12 & 7 \\
\hline \multirow[t]{3}{*}{5} & Number & 14 & 6 & 9 & 9 \\
\hline & Length & & 30 & 30 & 60 \\
\hline & Density & & 6 & 3 & 8 \\
\hline \multirow[t]{3}{*}{6} & Number & 13 & 6 & 6 & 9 \\
\hline & Length & & 30 & 60 & 60 \\
\hline & Density & & 6 & 12 & 8 \\
\hline \multirow[t]{3}{*}{7} & Number & 12 & 6 & 9 & 9 \\
\hline & Length & & 38 & 38 & 60 \\
\hline & Density & & 8 & 5 & 8 \\
\hline \multirow[t]{3}{*}{8} & Number & 7 & 6 & 6 & 9 \\
\hline & Length & & 38 & 60 & 60 \\
\hline & Density & & 8 & 12 & 8 \\
\hline \multirow[t]{3}{*}{9} & Number & 8 & 6 & 9 & 9 \\
\hline & Diameter & & 12 & 12 & 18 \\
\hline & Density & & 5 & 3 & 5 \\
\hline \multirow[t]{3}{*}{10} & Number & 4 & 6 & 6 & 9 \\
\hline & Diameter & & 12 & 18 & 18 \\
\hline & Density & & 5 & 8 & 5 \\
\hline \multirow[t]{3}{*}{11} & Number & 11 & 6 & 12 & 12 \\
\hline & Length & & 40 & 40 & 80 \\
\hline & Density & & 8 & 4 & 8 \\
\hline \multirow[t]{3}{*}{12} & Number & 9 & 6 & 6 & 12 \\
\hline & Length & & 40 & 80 & 80 \\
\hline & Density & & 8 & 16 & 8 \\
\hline \multirow{3}{*}{13} & Number & 5 & 6 & 12 & 12 \\
\hline & Diameter & & 18 & 18 & 27 \\
\hline & Density & & 8 & 5 & 8 \\
\hline \multirow[t]{3}{*}{14} & Number & 3 & 6 & 6 & 12 \\
\hline & Diameter & & 18 & 27 & 27 \\
\hline & Density & & 8 & 13 & 8 \\
\hline
\end{tabular}

Note: Diameter is given for trials with randomly placed objects and length is given for trials with lines. Values for lengths, diameters, and densities are reported in centimeters.

${ }^{a} 1$ is the trial in which number is most salient; 14 is the trial in which number is le ast salient. The degree of salience is based on data from pilot subjects. 
6-12), except there were two additional trials, each with 6 and 9 objects (trials 7 and 8) which were necessary in order to create a pair with equal densities using these numbers. (Trials 5 and 6 with 6 and 9 objects had the same length ratio as the other trials, so they could not have a pair with equal densities.) Within each set of four trials, there were two trials with lines of birds and two trials with birds placed randomly all over the cards. Within each of these sets of two trials, one trial had two arrays with the smaller number and one array with the larger number (e.g., 3,3 , and 6) and one trial had one array with the smaller number and two arrays with the larger number $(3,6$, and 6$)$. The ratio of the numbers was $1: 2$ for all trials except those with 6 and 9 objects. The ratio of the lengths and the densities of the lines of objects was also 1:2, except for trials 7 and 8 which had to be adjusted to create a pair with equal densities. On the trials with random configurations, the ratio of the diameters was 2:3 rather than $1: 2$ as in the length dimension, because the difference between the arrays was much too large with the latter ratio. The density of the displays with objects placed randomly was determined once the values were chosen for the number and area dimensions. The ratio of the densities was always between 1:2 and 2:3. The densities described in Table 1 are approximate for the trials with random placement because the densities between any two adjacent objects could not be exactly the same.

In the pretest, there were three blue poster board cards with a green square, a green triangle, or a purple triangle pasted on. Small plastic animals in a box were used in the counting post-test.

Conservation task. The stimuli for this task were intended to be similar enough to the attention task to avoid differences in performance due to stimulus differences, and yet different enough to avoid the children's unquestioning use of the same answers given in the first task a week earlier (see Table 2). Each trial had a standard blue card with birds identical to those of the attention task pasted on it and a blank blue card on which the birds were first placed down in correspondence to the birds on the standard cards and then transformed.

The conservation pretest examined the child's understanding of "same number" and "different number." There were two sets of cards with two cards in each set. One trial had two green squares on each card, but the cards differed in the orientation and placement of the squares. The other trial had one card with four red squares and one card with one red square.

\section{Procedure}

The children were individually tested in a small room in the school. The attention task was always given first to prevent any bias toward 
TABLE 2

Description of Stimuli on the Conservation Trials after Transformation

\begin{tabular}{clrr}
\hline Trial & Dimensions & Card 1 & Card 2 \\
\hline 1 & Number & 4 & 4 \\
& Diameter & 10 & 15 \\
Density & 5 & 10 \\
& Number & 4 & 4 \\
& Length & 20 & 40 \\
& Density & 6 & 12 \\
3 & Number & 8 & 8 \\
& Diameter & 14 & 21 \\
& Density & 5 & 10 \\
4 & Number & 8 & 8 \\
& Length & 45 & 67 \\
& Density & 6 & 9 \\
& Number & 8 & 8 \\
5 & Length & 35 & 70 \\
& Density & 5 & 10 \\
\hline
\end{tabular}

Note: Trials are listed in order of difficulty: 1 is the easiest, 5 is the most difficult.

Values for length, density, and diameter are reported in centimeters.

number responses which the conservation task might have created. The conservation task was given approximately 1 week later to avoid any carryover from the attention task.

Attention task. A standard color-form preference test served as a pretest for the verbal terms used in the attention task. Subjects were shown a green square, a green triangle, and a purple triangle and asked which two were most alike and why. Children who could not pick two cards which were similar and verbalize their preferences were rejected.

The attention task followed the pretest. The kindergarteners were randomly assigned to three conditions which differed in the order of the 14 trials: a different random order for each child, trials ordered from highest to lowest number salience, and trials ordered from lowest to highest number salience. The salience of number on each trial was defined by the number of children attending to number on each trial in pilot testing. All third-graders received the random condition. In each triad, no two cards of objects which agreed on one of the three dimensions, number, length or area, or density of objects, appeared in the same spatial positions more than two consecutive times. Cards with lines of objects were placed one above the other and cards with objects arranged randomly were placed in a pyramid (left, right, and centered above). It was necessary to use these two arrangements in order to keep 
all three arrays in front of the child; some of the linear arrays were quite long and some of the arrays with a random placement of objects were quite large. The subject was asked which two cards were most alike. He was also asked why he chose the two he did on the first two trials and every third trial thereafter. If a child indicated an irrelevant dimension (e.g., "they all have wings") he was told that that was true for all the cards. The question was repeated and if the child could not switch to a relevant dimension he was rejected. There were probes on the last trial. For the two pairs of cards not chosen, the child was asked, "Is there any way these two cards are alike? How are they alike?" Following the attention trials the children were asked to count out 12 toy animals from a box. Any children who could not do this were rejected.

Conservation task. Only kindergarteners were tested because the high level of conservation in kindergarteners indicated that it was not necessary to test the third-graders for conservation. On the two trials of the pretest, subjects were asked "Do both cards have the same number of squares or does one card have more squares than the other?" (On half the trials the order of the phrases was reversed.) The order of the trials was counterbalanced. Any child who was not correct was rejected. The pretest insured that the children understood the verbal terms and avoided a response bias of "same" since the answer on one trial was "one has more." Following the pretest, children were assigned to one of three conservation conditions, depending on the condition they had been in for the attention task. All children who received the trials in a random order in the attention task were also in the random condition in the conservation task. The only constraint on the random orders was that the first two trials could not be the two easiest or two hardest ones. Half of the subjects in the two ordered conditions of the attention task were placed in the same condition on the conservation task and half were given random orders. The order of difficulty of the conservation trials was based on data from pilot subjects.

For all five trials, children saw two identical arrays of objects. After the child agreed they had the same number, one of the arrays was either lengthened or spread out nonlinearly. Whether the transformed array or standard array was closer to the child was counterbalanced. The child was asked, "Do these have the same number of birds or does one of them have more?" and "How do you know?" The order of the two parts of the first question was alternated from trial to trial.

\section{Scoring}

Two criteria were used for scoring conservation responses. A conservation judgment $(C)$ was credited if the child stated that the arrays had the 
TABLE 3

Percentage of Number, Length or Area, and Density Responses FOR TYPES OF TRIALS ON THE ATTENTION TASK

\begin{tabular}{|c|c|c|c|c|c|c|}
\hline \multirow[b]{2}{*}{ Type of trial } & \multicolumn{2}{|c|}{$\begin{array}{l}\text { Number } \\
\text { responses } \\
(\%)\end{array}$} & \multicolumn{2}{|c|}{$\begin{array}{l}\text { Length or area } \\
\text { responses } \\
(\%)\end{array}$} & \multicolumn{2}{|c|}{$\begin{array}{c}\text { Density } \\
\text { responses } \\
(\%)\end{array}$} \\
\hline & $\begin{array}{l}\text { Kinder- } \\
\text { garteners }\end{array}$ & $\begin{array}{l}\text { Third- } \\
\text { graders }\end{array}$ & $\begin{array}{l}\text { Kinder- } \\
\text { garteners }\end{array}$ & $\begin{array}{l}\text { Third- } \\
\text { graders }\end{array}$ & $\begin{array}{l}\text { Kinder- } \\
\text { garteners }\end{array}$ & $\begin{array}{l}\text { Third- } \\
\text { graders }\end{array}$ \\
\hline \multicolumn{7}{|l|}{3 and 6 objects } \\
\hline Random & 80 & 100 & 12 & 0 & 8 & 0 \\
\hline Linear & 60 & 94 & 31 & 0 & 9 & 6 \\
\hline Total & 70 & 97 & 22 & 0 & 8 & 3 \\
\hline \multicolumn{7}{|l|}{6 and 9 objects } \\
\hline Random & 55 & 86 & 32 & 11 & 13 & 3 \\
\hline Linear & 20 & 72 & 67 & 17 & 13 & 11 \\
\hline Total & 38 & 79 & 49 & 14 & 13 & 7 \\
\hline \multicolumn{7}{|l|}{6 and 12 objects } \\
\hline Random & 74 & 94 & 19 & 6 & 8 & 0 \\
\hline Linear & 26 & 78 & 60 & 3 & 13 & 19 \\
\hline Total & 50 & 86 & 40 & 4 & 10 & 10 \\
\hline \multicolumn{7}{|l|}{ Total } \\
\hline Random & 70 & 94 & 21 & 6 & 9 & 0 \\
\hline Linear & 35 & 81 & 53 & 7 & 12 & 12 \\
\hline Total & 53 & 88 & 37 & 7 & 11 & 6 \\
\hline
\end{tabular}

same number after the transformation. The stricter criterion was a conservation judgement accompanied by an adequate explanation (EC), i.e., same number ("they both have four"), irrelevancy of transformation, addition/subtraction, compensation, previous equality, reversibility, and one-to-one correspondence. Only if the child did not count the objects after the transformation was "same number" considered adequate, because the child who counts probably does not think that conservation is logically necessary. The percentage of agreement between two raters with respect to whether an explanation was adequate was 95\%; agreement for type of adequate explanation was $93 \%$.

\section{RESULTS}

Since there were no sex differences on either task, all analyses were done with the sexes combined.

As predicted, the order of the trials significantly affected the percentage of number responses for the kindergarteners in the attention task, $F(2,83)=3.33, p<.05$. The most number responses were in the condition which began with trials in which number was most salient (55\%) and 
the least were in the condition with the opposite order $(42 \%)$. The random condition fell between the two (49\%). The frequency of responses to number on each of the trials in the random condition provided a check for the prearranged ordering of the trials in the other two conditions which was based on pilot testing. There was good replication: only 3 out of 14 trials were slightly out of place.

There was no effect of order of trials for the five trials on the conservation task. In addition, the set effect from the attention task did not carry over to the conservation task. That is, the three groups which saw the conservation trials in a random order but differed in the order in which they saw the attention trials showed no significant differences in conservation performance. In addition, children given the same order on both tasks did not differ significantly in their conservation performance.

It was desirable to have identical length ratios on all trials when analyzing the effect of number of objects and the type of array. Therefore, trials 7 and 8 of the attention task and trial 4 of the conservation task were omitted from the following analyses for these two variables.

As seen in Table 3, the sets of numbers used significantly affected the attention of kindergarteners, $F(2,170)=66.36, p<.001$ and third graders, $F(2,34)=3.95, p<.05$. For the kindergarteners, both the ratio and the magnitude of the two numbers were important. Scheffe post hoc comparisons indicated that the set 3 and 6 led to more number responses than 6 and $12(p<.05)$, and 6 and 12 led to significantly more number responses than the set 6 and $9(p<.05)$. The only significant difference for the third-graders was more number choices on trials with 3 and 6 objects than trials with 6 and 9 objects $(p<.05)$.

When the two arrays with the same number in a trial used the smaller (e.g., 3, 3, 6) rather than the larger (e.g., 3, 6, 6) of the pair, kindergarteners gave more number responses, $t(85)=3.29, p<.002$ ). Using the smaller pair gave $53 \%$ number responses and using the larger pair gave $44 \%$. There was no significant effect for the third-graders.

For kindergarteners, the two conservation trials with four objects were easier than the two which had eight, $t(85)=3.34, p<.001$ for $\mathrm{C}$ scores and $t(85)=3.40, p<.001$ for EC scores. There were conservation responses on $83 \%$ of the trials using four objects (67\% for $\mathrm{EC})$ and $73 \%$ of the trials using eight ( $56 \%$ for EC).

As expected, on the attention task, more number responses were given to arrays with objects placed randomly than those with objects arranged linearly, $t(85)=10.90, p<.001$ for the kindergarteners and $t$ (17) $=2.40, p<.05$ for the third-graders, see Table 3 . This effect was also significant for kindergarteners in the conservation task, $t(85)=4.02$, $p<.001$ for $\mathrm{C}$ scores and $t(85)=2.02, p<.05$ for EC scores. Kindergarteners gave conservation responses on $83 \%$ of the trials with random configurations ( $66 \%$ for EC) and $73 \%$ with linear ones ( $58 \%$ for EC). 
TABLE 4

Percentage of Number, Length or Area, and Density Responses on the attention Task for Each Subject Group

\begin{tabular}{lcccc}
\hline Subject group & $N$ & $\begin{array}{c}\text { Number } \\
\text { responses } \\
(\%)\end{array}$ & $\begin{array}{c}\text { Length or area } \\
\text { responses } \\
(\%)\end{array}$ & $\begin{array}{c}\text { Density } \\
\text { responses } \\
(\%)\end{array}$ \\
\hline Kindergarteners & 15 & 41 & 45 & 13 \\
$\quad$ Nonconservers & 35 & 46 & 41 & 12 \\
Transitionals & 36 & 54 & 38 & 9 \\
Conservers & 18 & 85 & 8 & 7 \\
Third-graders & & & & \\
\hline
\end{tabular}

In addition to the group analyses, individual performance is also of interest. Third-graders were clearly responding to the attention task on the basis of number ( 7 out of a possible 18 children responded only to number for all 14 trials and 17 had a majority of number responses). All but one kindergartener gave at least one number response but only three kindergarteners gave all number responses. Thirty of the 86 kindergarten children responded on the basis of number for more than half the trials and 26 responded to length and area on more than half the trials. While 50 kindergarteners gave density responses at least once, only one child responded on the basis of density for a majority of the trials. Similarly, on conservation trials, only four nonconservers said the shorter, denser row or bunch had more objects. Their numbers of density responses on the attention task were $0,0,3$, and 5 .

To examine more closely the relationship between performance on the attention task and the conservation task, there was a comparison of the attention of kindergarten nonconservers, kindergarten transitional conservers, kindergarten conservers, and third-graders (see Table 4). To be classified as a conserver, a child had to give both conservation answers and adequate explanations on all trials. The use of a strict criterion for conservation ensured that no transitional children were included in this group. Nonconservers gave no adequate explanations (but could give some conservation responses). All others were classified as transitionals. The groups differed significantly in the percentage of number responses, $F(3,100)=18.32, p<.001$, and length responses, $F(3,100)=13.85, p<.001$. There were no significant differences in density responses, which were uniformly low. Table 4 demonstrates that the differences in attention are in the expected direction. The progression from nonconservation in kindergarteners to conservation in thirdgraders is associated with a gradual increase in attention to number and a decrease in attention to length or area. 
The probe questions after the last trial revealed little ability to verbalize dimensions not responded to on the trial. Sixteen out of 41 kindergarteners could successfully answer the probe for number, 8 out of 20 for length, 1 out of 33 for area, and 2 out of 68 for density. For the third-graders, the three children who did not respond on the basis of number for the last trial could verbalize it when probed, 5 out of 10 thirdgraders could verbalize length, 2 out of 6 could verbalize area, and 1 out of 17 third-graders could verbalize density.

\section{DISCUSSION}

The results indicate a close relationship between attention to number and number conservation. First, there was an increase in attention to number from kindergarten nonconservers to kindergarten transitional conservers to kindergarten conservers to third-graders. Nonconservers, as a group, attended equally to number and length or area. Kindergarten conservers attended to number more than length and thirdgraders usually attended to number. Second, a small number of objects and arrays with random placement of objects rather than lines facilitated both attention to number and conservation of number.

The developmental relationship between attention to number and conservation may be an example of a model described by Flavell and Wohlwill $(1969$, p. 89$)$, in which "a child begins to make progress on one of ... two acquisitions, his progress helps to mediate a beginning on the other, and so on back and forth, leading eventually to the mastery of both." Since there is a good deal of attention to number in nonconservers on the easier trials, this attention apparently does not require the ability to conserve. In fact, all but one nonconserver attended to number on at least one trial. This early attentional ability, then, may aid in the development of conservation by helping the child select out the number dimension, keep track of the number dimension over time and events (the transformation), and resist other potentially distracting dimensions such as length. Thus, attention to number may be necessary, but certainly not sufficient, for conservation of number. Conversely, the development of conservation may encourage attention to number by developing a quantitative set in the child's approach to problems. With his growing experience with quantifying the world and, consequently, the generalization and stabilization of his conservation ability, his attention to number becomes even stronger. Thus, third-graders attend to number more than kindergarten conservers. This reciprocal influence of attention and conservation points toward an extended period of development for conservation.

When the results of the present study are compared with other studies, it is clear that stimulus variables are of great importance. Henry (1971) 
also found a developmental increase in attention to number but the overall level was much lower. For example, his kindergarten nonconservers attended to number only $4 \%$ of the time. The difference is probably due to the fact that all of Henry's attention trials had rows of 7 and 14 objects, which may have increased attention to length. Some of the trials in the present study had a smaller number of objects and random as well as linear arrays. Miller (1973) found very little attention to liquid quantity among either kindergarten nonconservers or conservers. Miller's attentional preference task included transformations which may have created a more complex situation than the static presentation in the present study. This complexity may have made it more difficult to attend to quantity. Or, it simply may be more difficult to attend to liquid quantity than number.

The children's performance on the number tasks in the present study was greatly influenced by the stimulus variables: number of objects, ordering of objects (initial trial with number most salient or initial trial with number least salient), and configurations of objects. Thus, whether a child appears to be a conserver or nonconserver and whether he attends to number depends in part on the situation. This supports Zimiles' (1966) and Gelman's (1969) suggestion that children have a multidimensional definition of number (e.g., length, density, and true number), and which definition is used depends on many situational factors.

The effect of the stimulus variables may come from their influence on the child's choice of what Gelman (1972) calls estimators, cognitive processes used to determine quantity, such as the number of objects in a set. Lines and large numbers of objects may lead the child to use salient perceptual dimensions, e.g., length, as estimators or, in the attention task, even discourage him from trying to estimate number at all; it is easier to simply respond to length. In contrast, random arrays and small numbers elicit other, more reliable, estimators such as subitizing (the direct perception of small numbers without counting), counting, use of one-to-one correspondence, etc. The smallest arrays in the present study fall within the number of objects that young children can subitize (Chi \& Klahr, 1975). In addition, young children can count a small number of objects more easily than a larger number. This superior performance with small numbers is consistent with past research (e.g.. Baron et al., 1975; Gelman, 1972; Lawson et al., 1974; Smither et al., 1974). With the random displays, where no perceptual cues are especially salient and the arrays are too large to subitize, counting may be used. This line of reasoning is consistent with recent work by Klahr and Wallace (1973) and Schaeffer, Eggleston, \& Scott (1974). Gelman points out that the child's choice of an estimator influences whether he uses operators (cognitive processes by which a child decides if a transformation affects the quantity of a set of objects, as in the conservation task). 
In short, it may be that the stimulus situation (e.g., what is salient) affects attention which affects the choice of an estimator (or even whether to try to estimate number), which in turn affects conservation performance.

The effect of the order of presentation of the trials on the attention task suggests that when the trials in which number is more salient are first, they create a set for number. This set prevents the child from attending to features which he would attend to if the trials in which number is less salient were presented first. Baron et al., (1975) reported that beginning with trials with large numbers of objects caused more biases towards length when estimating number and length than beginning with small numbers. Miller et al., (1975) found a set effect with conservation trials. The lack of a set effect in the conservation task in the present study was probably due to the small number of trials (five).

The rarity of responses to density is consistent with other research (Gelman, 1972; Henry, 1970). This result raises questions about the role of compensation in conservation of number. According to Piaget, the knowledge that changes in density and length are compensatory is one of the underlying bases of conservation. If children are in fact aware of density, it clcarly is not a salient dimension. This finding is analogous to the rarity of width responses in Miller's (1973) study of liquid quantity.

In order to achieve a clearer picture of the relationship between attention and conservation, it will be necessary to relate developmental changes in attention and the development of various conservations. The number, length, and substance conservations typically develop early in the concrete operational period. Length is a dimension in each of these tasks, but is relevant for length and misleading for number and substance conservation. In the present study, an increase with age was associated with an increase in attention to number and a decrease in attention to length. This was interpreted as evidence for the development of a quantitative set. However, length is an important dimension in some situations and attention to length would be expected to increase as conservation of length develops. It is possible that as attention to number increases and attention to length decreases during development, the development of conservation of length is temporarily impaired. There does, in fact, appear to be a small décalage between number and length conservation (e.g., Goldschmid, 1967). Alternatively, it may simply be that attention is different for a line of discrete objects with spaces between them and an unbroken line, such as a pencil or stick.

The present study and related studies indicate that a full description of the development of conservation will include various subdevelopments such as earlier number abilities and attentional processes. Further research should identify the causes and effects underlying the rclationships found in the present study. 


\section{REFERENCES}

Baron, J., Lawson, G., \& Siegel, L. S. Effects of training and set size on children's judgments of number and length. Developmental Psychology, 1975, 11, 585-588.

Chi, T. H., \& Klahr, D. Span and rate of apprehension in children and adults. Journal of Experimental Child Psychology, 1975, 19, 434-439.

Flavell, J. H., \& Wohlwill, J. F. Formal and functional aspects of cognitive development. In D. Elkind \& J. H. Flavell (Eds.), Studies in cognitive development. New York: Oxford University Press, 1969.

Gelman, R. Conservation acquisition: A problem of learning to attend to relevant attributes. Journal of Experimental Child Psychology, 1969, 7, 167-187.

Gelman, R. The nature and development of early number concepts. In H. W. Reese (Ed.), Advances in child development and behavior (Vol. 7). New York: Academic Press, 1972.

Goldschmid, M. L. Different types of conservation and nonconservation and their relation to age, sex, IQ, MA, and vocabulary. Child Development, 1967, 38, 12291246.

Klahr, D., \& Wallace, J. G. The role of quantification operators in the development of conservation of quantity. Cognitive Psychology, 1973, 4, 301-327.

Lawson, G., Baron, J., \& Siegel, L. The role of number and length cues in children's quantitative judgment. Child Development, 1974, 45, 731-736.

Miller, P. H. Attention to stimulus dimensions in the conservation of liquid quantity. Child Development, 1973, 44, 129-136.

Miller, P. H., Heldmeyer, K. H., \& Miller, S. A. Facilitation of conservation of number in young children. Developmental Psychology, 1975, 11, 253.

Schaeffer, B., Eggleston, V. H., \& Scott, J. L. Number development in young children. Cognitive Psychology, 1974, 6, 357-359.

Smither, S. J., Smiley, S. S., \& Rees, R. The use of perceptual cues for number judgment by young children. Child Development, 1974, 45, 693-699.

Suchman, R. G. Color-form preference, discrimination accuracy and learning of deaf and hearing children. Child Development, 1966, 37, 439-451.

Whiteman, M., \& Peisach, E. Perceptual and sensorimotor supports for conservation tasks. Developmental Psychology, 1970, 2, 247-256.

Winer, G. Conservation of different quantities among preschool children. Child Development, 1974, 45, 839-842.

Zimiles, H. The development of conservation and differentiation of number. Monographs of the Society for Research in Child Development, 1966, 31 (6, Serial No. 108).

\section{REFERENCE NOTES}

1. Henry, D. Attentional and cardinal-ordinal factors in the conservation of number. Unpublished doctoral dissertation, University of Minnesota, 1971.

ReCEIVED October 21, 1975; REvised April 15, 1976. 